\title{
Topology-guided Visualization of Constrained Vector Fields
}

\author{
Ronald Peikert and Filip Sadlo \\ Computer Graphics Laboratory, Computer Science Department, \\ ETH Zurich, Switzerland \{peikert, sadlo\}@inf .ethz.ch
}

In this study we explore ways of using precomputed vector field topology as a guide for interactive feature-based visualization of flow simulation data. Beyond streamline seeding based on critical points, we focus mainly on computing special stream surfaces related to critical points and periodic orbits. We address the special case of divergence-free vector fields which is often met in practical CFD data, and we extend the topological analysis to no-slip boundaries by treating $3 \mathrm{D}$ velocity and $2 \mathrm{D}$ wall shear stress in a unified way. Finally we apply the proposed techniques to flow simulation data and demonstrate their usefulness for the purpose of studying recirculation and separation phenomena.

\section{Introduction}

Vector field topology as a means to visualize the structure of fluid flow has been introduced by Helman and Hesselink [HH89]. A first generation of topology-based visualization methods locates, classifies, and displays critical points of the given vector field as point icons. Sophisticated icons can convey various information on the local topology and geometry of the flow [GLL91]. Beyond critical points, periodic orbits can be located [WS02] and classified based on their Poincaré maps. Another use of Poincaré maps is to include them into 3D visualizations for a better understanding of the flow near the periodic orbit [LKG98]. Finally, the topological skeleton of the vector field is obtained by computing all critical points and periodic orbits together with their stable and unstable manifolds, i.e. the union of streamlines converging in positive or negative time to the critical point or periodic orbit.

The striking property of these direct topological methods is that they are fully automatic and free of tuning parameters. A practical limitation is however that for many kinds of vector field data the topology is far too rich to be displayed in full detail. This led to concepts such as topological simplification [LL99, TSH00]. The stable or unstable 2D manifold of a 3D saddle point is a particularly interesting feature as it indicates a local flow separation. However, displaying a larger number of such stream surfaces leads to occlusion problems. Again, simplification is needed, 
and a possible solution is to only display their intersection curves, known as saddle connectors [TWH*03] or as heteroclinic and homoclinic orbits.

When considering the use of vector field topology for visualizing CFD data, it has to be kept in mind that topological features are not the final result an engineer or scientist wants to see. The topological analysis can, however, be a valuable first step to be followed by other visualization techniques. One possible strategy is to use topology for segmenting a vector field into regions of similar flow. This is particularly successful in 2D, while in 3D the notion of segmentation must be somehow relaxed to a more local property [MBS*04].

A second approach is to use topological features as guides for a different type of visualization. For example, a region-of-interest can be defined or a set of streamlines can be seeded [YKP05] based on topological features. If an interactive, explorative type of visualization is pursued, visual clutter can usually be avoided, so that simplification is often not needed, even when stream surfaces are used for the visualization.

In this work we focus mainly on 2D manifolds of 3D saddles and saddle type periodic orbits. We believe that compared to arbitrarily chosen stream surfaces, such 2D manifolds can be more expressive and in most cases also of a simpler shape. In particular, recirculation zones and separation surfaces are well suited for this type of visualization. The underlying idea of visualizing topologically meaningful stream surfaces and their relationship to topological features has previously been used by Garth et al. [GTS*04] in their visualization of a vortex breakdown in the flow over a delta wing.

Computing stream surfaces in the vicinity or even converging to singularities, requires robust algorithms. The classical stream surface algorithm is that of Hultquist [Hu192]. Here, the stream surface is generated by integrating a sequence of discretized streamlines and triangulating between them where appropriate. Triangle shape is optimized by choosing the shorter of the two possible edges in the process of triangulating between two streamlines. Triangle size is controlled by seeding new streamlines or stopping streamlines. This basic algorithm can be implemented with a depth-first strategy. However, to evaluate the criteria for adding or stopping a streamline, it is more convenient to used a breadth-first strategy where a current "front" is used. Garth et al. [GTS*04] added a refinement criterion based on the angle between adjacent segments of the front. Theisel et al. [TWH*03] remarked that Hultquist's algorithm fails if the tangents of the front are almost in the direction of the vector field, a situation which can arise e.g. near critical points or periodic orbits. They use as an initial front a line perpendicular to the vector field. This way, even tightly spiralling streamlines can be handled. However, the choice of the line is critical to avoid cracks or multiple coverings. Also, this approach produces spurious internal boundaries which have to be postprocessed for a correct result.

Vector field topology requires differentiable 2D or 3D vector fields. Usually, no further restriction is made for the vector fields. This is appropriate in the context of dynamical systems [GH83], which was the original application of vector field topology. Vector fields arising in physics, however, are often known to be divergencefree or irrotational or both. In Sec. 2 we will explore some of the implications of zero divergence to vector field topology and its application to the visualization of 
flow structures. In most CFD simulations, no-slip boundary conditions are imposed on some of the boundaries. Extending vector field topology to no-slip boundaries is the topic of Sec. 3. And finally, in Sec. 4 we will discuss some applications.

\section{Topology of divergence-free vector fields}

The case of a divergence-free (sometimes called solenoidal) vector field is particularly important in fluid dynamics. Examples of divergence-free vector fields are: velocity fields in hydrodynamics, vorticity fields, magnetic fields. Further divergencefree fields may be obtained by multiplying a given vector field (having neither sources nor sinks) with an appropriate scalar field. This is based on the fact that multiplication with a nonvanishing scalar field does not change the topology. As an example, the momentum field has the same topology as the velocity field, because they are identical up to a nonvanishing factor, the density. If the velocity field for instance is a steady solution of the compressible continuity equation $\frac{\partial \rho}{\partial t}+\nabla \cdot(\rho \mathbf{u})=0$, then the momentum field would be divergence-free.

The special case of divergence-free vector fields has an effect on the analysis of critical points. Asimov [Asi93] mentions that in 2D and 3D divergence-free vector fields sources and sinks are not possible, but any types of saddles are. And in the 2D case, there is a new structurally stable type of critical points, namely the center. The center is said to have constrained structural stability. The center has the property that in a neighborhood, all streamlines are closed.

A similar analysis as for critical points can be done for periodic orbits (closed streamlines) in divergence-free 3D vector fields. Periodic orbits are of interest as they can indicate recirculation zones. Many properties of the periodic orbit can be studied in two dimensions by computing a Poincaré map. This is done by selecting a surface patch $\mathscr{S}$ which is everywhere transversal to the vector field. If sufficiently small, this so-called Poincaré section $\mathscr{S}$ is intersected by the periodic orbit in a single point. For a sufficiently close point $\mathbf{x} \in \mathscr{S}$, the Poincaré map $\mathscr{P}(\mathbf{x})$ is then defined as the first intersection of the streamline seeded at $\mathbf{x}$ with $\mathscr{S}$.

Periodic orbits are called hyperbolic if the eigenvalues of the linearization $\mathbf{P}$ of $\mathscr{P}$, the so-called Floquet multipliers, lie off the complex unit circle. According to Asimov [Asi93], hyperbolic periodic orbits can be classified into sources, sinks, saddles, twisted saddles, spiral sources and spiral sinks depending on the Floquet multipliers.

The Poincaré map $\mathscr{P}$ has a fixed point where it is intersected by the periodic orbit. For the eigenvalue analysis, $\mathscr{P}$ is now linearized in a neighborhood of a fixed point. This linearized map $\mathbf{P}$ takes an infinitesimal circle centered at the fixed point to an ellipse with the same center. If the velocity field is divergence-free and thus volume preserving, the fluxes through the circle and the ellipse are equal. The flux is the integral of the normal velocity over the circle or ellipse. The normal velocity can be linearized as well, and because of symmetry, it can be replaced by its average. It follows that $\mathbf{P}$ must be area conserving, i.e. has a determinant of one. The sign is positive because a Poincaré map always conserves orientation. 


\subsection{Source and sink periodic orbits}

It is now easy to see that periodic orbits of type source or sink are not possible for a divergence-free vector field. In the case of a source (either node source or spiral source), both eigenvalues lie outside of the complex unit circle. Hence, the determinant of $\mathbf{P}$ has absolute value greater than one, meaning that the area of an infinitesimal circle is not conserved under $\mathscr{P}$. The same can be concluded for sinks.

\subsection{Saddle and twisted saddle periodic orbits}

Periodic orbits of type saddle or twisted saddle are possible in divergence-free vector fields. Such periodic orbits are particularly suitable for visualization because they have a stable and an unstable manifold which are stream surfaces converging to the periodic orbit in positive or negative time. The nice property of these manifolds is that they "return to themselves" when following the periodic orbit for a full turn. This means, if a streamline is seeded on the intersection of the manifold with a Poincaré section and sufficiently close to the periodic orbit, it will return to the same intersection curve. If the seed curve is reduced to an infinitesimal line segment, its behavior is given by the eigenvalues of $\mathbf{P}$. If both eigenvalues are positive, the generated stream surface band returns untwisted to the Poincaré section. It may have done an integer number of full (360 degrees) so-called extrinsic twists. And it can shrink or stretch, depending on the eigenvalue associated to the eigenvector aligned with the seed line. If both eigenvalues are negative, the stream surface band does an additional half twist. In our case of divergence-free vector fields the product of the two eigenvalues equals one because of the above-mentioned conservation of area. Because of their property to return to the seed curve, (un-)stable manifolds are the ideal stream surfaces to depict the local behavior of the field near the periodic orbit.

\subsection{Center periodic orbits}

If a periodic orbit in a divergence-free vector field has complex eigenvalues of $\mathbf{P}$ its type can be neither spiral source nor spiral sink. It must be the in-between case with eigenvalues on the complex unit circle. This is not a hyperbolic case, but has the constrained structural stability similar to that of center critical points in 2D fields. By analogy, we call it a center periodic orbit.

The linearized Poincaré map $\mathbf{P}$ of such a periodic orbit has complex eigenvalues and a determinant of one. It can therefore be written as $\mathbf{P}=\mathbf{T R T}^{\mathbf{1}}$ where $\mathbf{R}$ is a pure rotation. It follows that $\mathbf{T}$ applied to an infinitesimal circle is an ellipse which is invariant under $\mathbf{P}$. This means that a stream surface seeded at this ellipse returns to the ellipse after following the periodic orbit for a full turn. The same idea can be used for finding finite invariant tori. The goal is here to find a closed seeding curve in the Poincaré section which is invariant under $\mathscr{P}$. As an initial guess a scaled version of the infinitesimal ellipse can be used. If starting from this an invariant seeding curve can be found, the problem is solved. However, we found that in practice this is a numerically challenging problem. 


\section{Topology near no-slip boundaries}

\subsection{Velocity and wall shear stress}

By definition, a critical point is an isolated singularity of the vector field. Vector topology does not treat extended singularities. However, these occur in practical vector fields having solid boundaries with associated no slip boundary conditions. The velocity field $\mathbf{u}(\mathbf{x})$ itself is zero on such a boundary, but by using the unsigned distance to the boundary as a scalar field $s(\mathbf{x})$, it can be written as a product

$$
\mathbf{u}(\mathbf{x})=s(\mathbf{x}) \tilde{\mathbf{u}}(\mathbf{x})
$$

where the vector field $\tilde{\mathbf{u}}(\mathbf{x})$ can be assumed to exist also on the boundary and to be nondegenerate there.

From the divergence-free criterion follows for points on the boundary:

$$
0=\nabla \cdot(s \tilde{\mathbf{u}})=(\nabla s) \cdot \tilde{\mathbf{u}}+s(\nabla \cdot \tilde{\mathbf{u}})=(\nabla s) \cdot \tilde{\mathbf{u}}
$$

which means that on the boundary the field $\tilde{\mathbf{u}}$ has no normal component. In terms of vector field topology this means that no streamline of $\tilde{\mathbf{u}}$ ever passes from the solid boundary to the interior or vice versa.

If Eq. 2 holds, then on the boundary, $\tilde{\mathbf{u}}$ is related to the wall shear stress $\tau_{w}$ by $\tau_{w}=\mu \tilde{\mathbf{u}}$ where $\mu$ is the kinematic viscosity of the fluid. Because of this proportionality $\tilde{\mathbf{u}}$ has the same topology as the 2D field of wall shear stresses. At interior points, $s$ is nonzero and therefore $\tilde{\mathbf{u}}$ has the same topology as $\mathbf{u}$. Hence, the field $\tilde{\mathbf{u}}$ nicely combines the wall shear field with the interior velocity field. However, this relies on the divergence-free property of the vector field. In the general case the field $\tilde{\mathbf{u}}$ has a normal component on the solid boundary. It can not be used to produce the topology of both the velocity field and the wall shear field. Of course the two vector fields could be blended, but then the topology of $\mathbf{u}$ may not be conserved.

Returning to the case of a divergence-free field, we saw that no streamline of ú passes from the interior to the boundary. But there may be convergence towards critical points on the solid boundary, which are 3D saddle points having two of its eigendirections along the boundary surface. Also, convergence towards periodic orbits on the boundary is possible.

The advantage of using the field $\tilde{\mathbf{u}}$ is that it is no more necessary to extract both $2 \mathrm{D}$ and $3 \mathrm{D}$ critical points (with possible consistency issues). Critical points on the boundary are now regular 3D critical points. In the special case of $\mathbf{u}$ being divergence-free, sources and sinks can be excluded due to structural stability and the fact that $\tilde{\mathbf{u}}$ has the same topology as $\mathbf{u}$. Consequently, such critical points must be saddles or spiral saddles. Furthermore, by Eq. 2 their two-dimensional stable or unstable manifolds lie completely on the boundary. The eigenvalue belonging to the remaining eigenvector is real-valued. Its sign determines whether the point is on a separation line (positive sign) or a reattachment line (negative sign).

In discrete data, dividing by $s$ has the drawback that due to interpolation inside the cells the topology is changed. A better strategy is to use the original field $\mathbf{u}$ for 
computing and analyzing the critical points in all cells which are not adjacent to no-slip boundaries. Only for computing the topology in the first layer of cells at the boundary, the modified field $\tilde{\mathbf{u}}$ is actually needed. The following steps are performed for cells adjacent to no-slip boundaries:

1. On interior nodes: compute $\tilde{\mathbf{u}}$ by dividing $\mathbf{u}$ by the wall distance.

2. On boundary nodes: interpolate $\mathbf{u}$ on two points on the boundary normal, compute $\tilde{\mathbf{u}}$, and linearly extrapolate to the boundary node.

3. Find critical points on the cell faces on the no-slip boundary. Use a 2D algorithm for finding the critical points, but classify them as 3D critical points.

\subsection{Critical points on no-slip boundaries}

Critical points on no-slip boundaries are important features for the study of flow separation. By applying the 3D classification, we will now concentrate on saddles and spiral saddles and ignore sinks and sources. These are of minor interest for the study of flow separation, and in divergence-free vector fields they do not occur.

Attracting (i.e. 2:1) saddles and spiral saddles have their stable manifold completely on the boundary. Any boundary curve of this manifold is a separation line. Similarly, repelling (1:2) saddles and spiral saddles have their unstable manifold on the boundary, so any boundary curve of it is a reattachment line. The case of spiralling separation (called tornado-type separation in [SGH06]) has not been discussed much in the visualization community.

A pattern we encountered often consists of a pair of spiral saddles, one of them in the interior and one on a solid boundary (see Fig. 1, points $C_{1}$ and $C_{2}$, respectively). They are rotating in the same sense and mark a recirculation area.

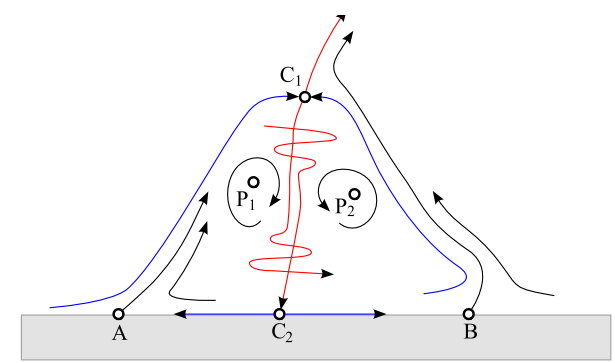

Fig. 1. Sketch of typical recirculation zone with two critical points of type spiral saddle $\left(C_{1}, C_{2}\right)$ and one periodic orbit $\left(P_{1}, P_{2}\right)$ involved. 1D manifolds (red curves) nearly meet. 2D manifolds (shown as blue curves) have a strong spiralling component.

The 1D manifolds nearly meet, while the 2D manifold of $C_{1}$ encloses the recirculation zone. This stream surface is not closed, so recirculation is not perfect. Within the recirculation zone there is a periodic orbit $\left(P_{1}\right.$ and $\left.P_{2}\right)$. Finally, the points $A$ and $B$ appearing as saddles in the planar section, seem to indicate a separation line. However, these points are topologically nothing special, they are just the points where the 
skin friction line is intersected orthogonally by the planar section. This means that many nearby skin friction lines can be regarded as separation lines.

Surana et al. in their exact theory of flow separation [SGH06] suggest as a criterion "strong hyperbolicity", i.e. large absolute eigenvalues of the saddles in the orthogonal section. An alternative and purely topological definition would be to pick the boundary curve of the unstable manifold of $C_{2}$ (which lies entirely on the solid boundary). In general this is composed of separatrices of nearby saddle points on the solid boundary.

\section{Applications}

\subsection{Pelton turbine}

A first application is a dataset resulting from a CFD simulation by VA Tech Hydro for the study of a Pelton turbine with the primary goal to optimize the stability of the water jets. The jets generated in the injectors (Fig. 2) must have a temporally stable circular cross section in order to optimally hit the runner buckets. Quality of the jets is mainly affected by vortices evolving in the outer ring where the water is deflected into the injectors. In Fig. 3 taken near the first of six injectors, the red stream surface shows the separation vortex arising because the flow does not follow the boundary. The yellow stream surface shows a smaller scale tornado-type separation.

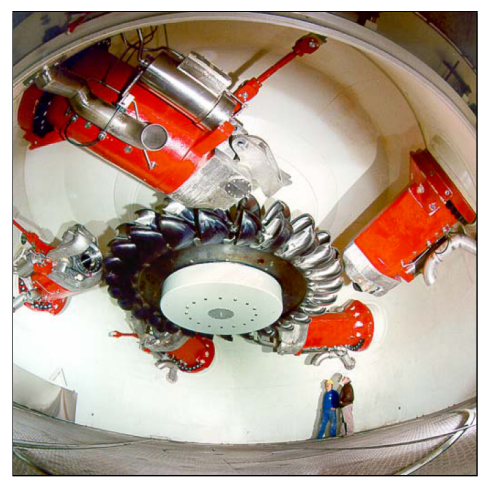

Fig. 2. Pelton turbine with five injectors.

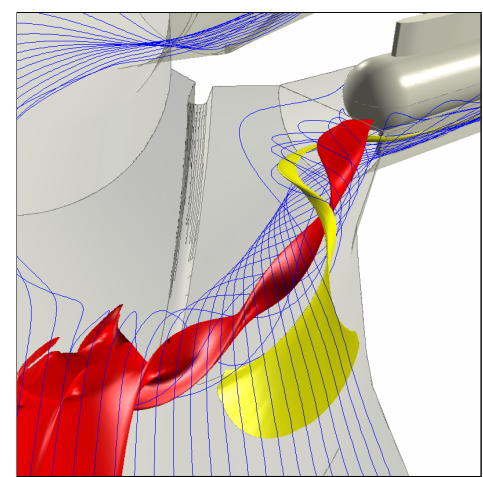

Fig. 3. Two vortices extending from the ring distributor into the first (of six) injectors

Inspecting the nearby critical points reveals that there is a pair of spiral saddles in this region, one of them is on the no-slip boundary (upper right in Fig. 4). A quick exploration by integrating a streamline forward and backward from seed points near the critical points gives an idea of the stable and unstable manifolds of the two spiral saddles (Fig. 5). 


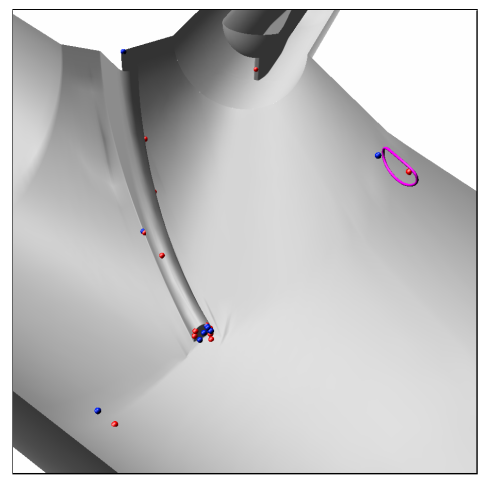

Fig. 4. Extracted interior (blue) and boundary (red) critical points. Periodic orbit (magenta).

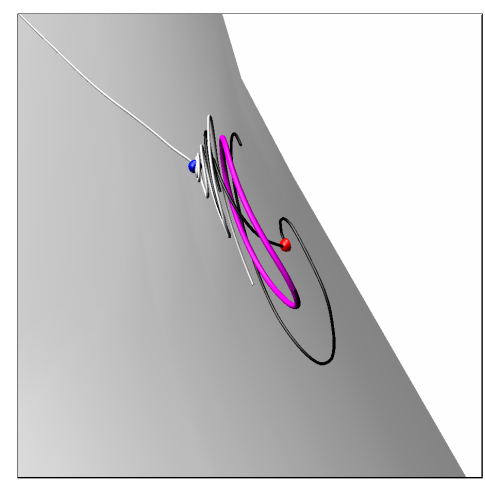

Fig. 5. Streamlines seeded near the boundary critical point (black) and the interior critical point (white).

Consistent with the situation sketched in Fig. 1, the stable manifold of the interior critical point encloses the recirculation zone (Fig. 6). The recirculation zone contains a single periodic orbit which is of twisted saddle type (Fig. 7). In this case, the stable and unstable manifolds of the periodic orbit are classical Möbius strips with a half twist and no further extrinsic twisting.

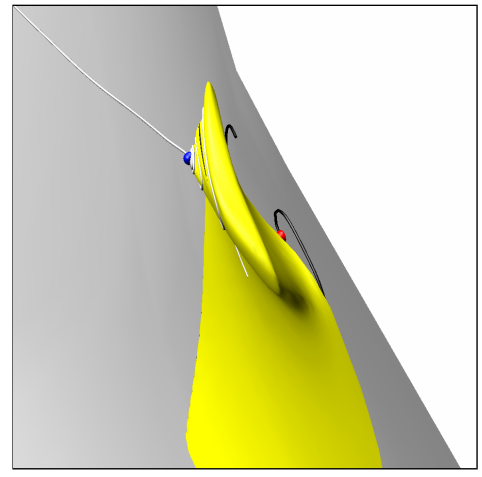

Fig. 6. Stable manifold of interior critical point.

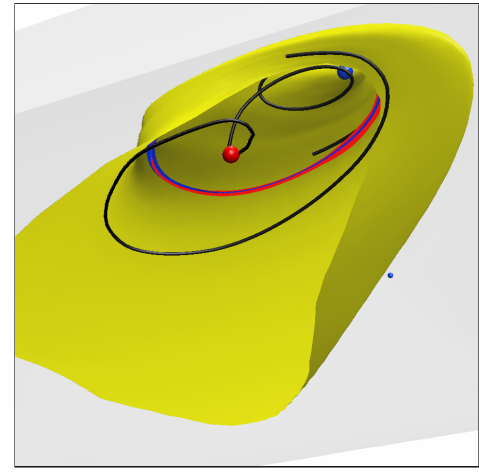

Fig. 7. View from the wall with stable and unstable manifolds of the periodic orbit (red and blue stream surfaces).

Similar flow patterns as near the first injector also appear near the third and fifth injector. In all cases, a periodic orbit of type twisted saddle can be observed. However, in the case of the third injector, the eigenvalues are relatively close to -1 , which suggests that instead of the twisted saddle, the center type (with a rotation angle close to 180 degrees) would be possible as well for slightly different data. 


\subsection{Draft tube}

As a second application, we examined the flow in the incompressible CFD simulation of a Francis draft tube. The design of the draft tube is such that in its lower part it is split into two channels. As observed in the simulation data, the right channel exhibits significantly stronger vortices. For topological interests we picked one of the strong vortices extending horizontally and almost orthogonally to the primary flow direction. The transient simulation of this vortex consists of 3 interesting and quite steady phases: First there is a vortex breakdown bubble of the unstable 2D manifold of a spiral saddle, enclosed in the stable 2D manifold of a spiral saddle. Then the bubble collapses and the stable manifold develops into a vortex breakdown bubble. Finally this bubble collapses too, leaving a common vortex. We have chosen a time step of the first phase where the vortex breakdown bubble is quite steady and hence the examination of its instantaneous topology should reveal some of its properties. Additionally, as reported by [SMH98], vortex breakdown bubbles are not necessarily the result of unsteady flow behavior.

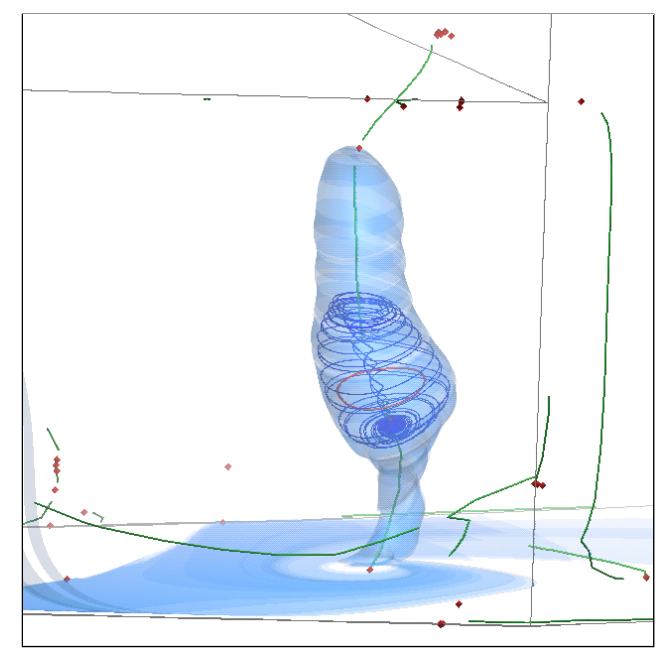

Fig. 8. Tornado-type separation and vortex in the draft tube dataset. Stream surface (transparent blue) starts at saddle and goes upstream enclosing a vortex breakdown bubble (blue streamline) containing a periodic orbit (red). Critical points (red) and vortex core lines (green).

Fig. 8 gives a view from top on the flow going from left to right. It shows (from bottom to top) a tornado-type separation with a critical point on the (outer) wall, and a vortex core line that connects to that critical point and extends across the channel into the part where the two channels merge. There is a recirculation region identified as a vortex breakdown bubble with a critical point at its bottom and a periodic orbit inside it. Another critical point resides above the bubble where the detected core line is disrupted. The stable 2D manifold of that saddle is visualized by an upstream surface that encloses the vortex breakdown bubble and approaches the wall. 


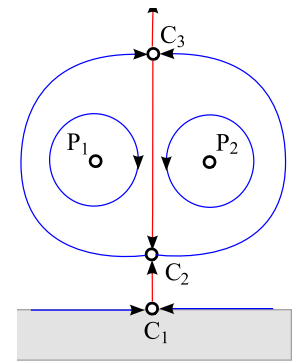

Fig. 9. Sketch of ideal (unperturbed) vortex breakdown bubble. Critical points $\left(C_{1}, C_{2}, C_{3}\right)$ and one periodic orbit $\left(P_{1}, P_{2}\right)$.

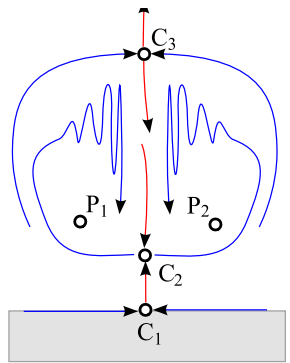

Fig. 10. Sketch of real-world (perturbed) vortex breakdown bubble. No intersection of the two 2D manifolds due to nonzero divergence in this region.

Unperturbed vortex breakdown bubbles (Fig. 9) are axisymmetric and consist of nested invariant tori. In the real world [Ven02, TH03], they contain regions of chaotic dynamics with possible islands of stability and KAM tori (impermeable) or Cantori (permeable) separating the regions (Fig. 10). We refer the reader to the paper of Sotiropoulos et al. [SVL01] for details. In the field of visualization, vortex breakdown bubbles have been studied recently ([TGK*04, GTS04, GTS*04]).

In our case, a vortex breakdown bubble containing a periodic orbit has been identified (Fig. 10). It seems that the stable 2D manifold of the upper saddle (transparent stream surface in Fig. 8) marks the end of the recirculation region since Spohn et al. [SMH98] report that vortex breakdown bubbles exhibit permanent inflow and outflow at the downstream tail. The fact that the two manifolds do not intersect is due to the inaccuracy of the simulation leading to nonzero divergence in this region.

Computing the vortex breakdown bubble as a stream surface seems impossible with Hultquist-type algorithms due to the complex folding and also due to the quasiperiodicity of the streamlines. Since a single streamline covers the toroidal stream surface densely, it can be seeded near the critical point and sampled on a voxel grid. The resulting field can then be visualized by an isosurface. To reduce aliasing effects and enhance resolution, a voxel value is not set in a binary manner when the streamline passes but computed based on coverage. An initial sequence of integration steps was not sampled in order to avoid an isolated spiral from the saddle point to the unstable manifold of the bubble.

Fig. 11 shows a slice of the resulting voxel field after tracing the particle for $10^{9}$ time steps. Its resolution is $750 \times 600 \times 600$ and it spans the complete bubble. Fig. 12 shows a finer sampling of a subregion. This makes the massive folding of the surface visible. Fig. 13 and 14 show an isosurface of the voxel field with the complete bubble. The isolevel was chosen to be $5 \%$ instead of $50 \%$ in order to avoid unmanageably many triangles in the fine folds. By adding a Gaussian smoothing step, we were able to cut down the the triangle count to about 20 million. 


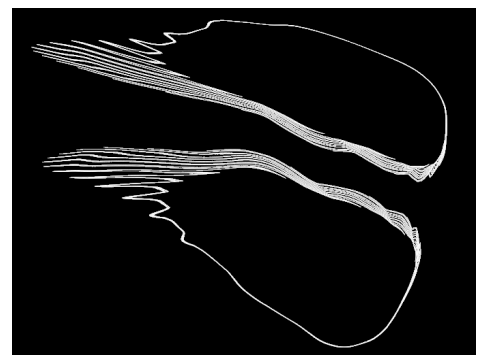

Fig. 11. Slice of the voxel field that sampled a single streamline.

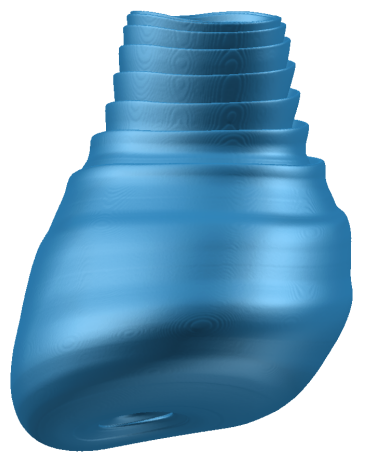

Fig. 13. Isosurface of the voxel-sampled single streamline on the surface of the breakdown bubble.

\section{Conclusion}

We gave examples of flow features in real CFD datasets which can be nicely illustrated by $2 \mathrm{D}$ manifolds of 3D saddles. Our experience showed that stream surface integration gets particularly challenging for these special cases of stream surfaces. Very robust stream surface algorithms are required which can cope with situations such as tightly winding spirals occurring in 2D manifolds of spiral saddles or saddle type periodic orbits. This issue is worth being addressed in further work, and as the ultimate goal in this line of research we see a stream surface algorithm which is fully "topology aware", i.e. which behaves correctly when integration approaches any kind of topological feature. The authors acknowledge VA Tech Hydro for the simulation data. This work was funded by Swiss Commission for Technology and Innovation grant 7338.2 ESPP-ES. 


\section{References}

[Asi93] Asimov, D.: Notes on the Topology of Vector Fields and Flows. Tech. Report RNR93-003, NASA Ames Research Center (1993)

[GH83] Guckenheimer,J., Holmes, P.: Nonlinear Oscillations, Dynamical Systems and Bifurcations of Vector Fields. Springer, New York (1983)

[GLL91] Globus, A., Levit, C., Lasinski, T.: A tool for visualizing the topology of threedimensional vector fields, In Proc. IEEE Visualization 91, 33-40 (1991)

[GTS*04] Garth, C., Tricoche, X., Salzbrunn, T., Bobach, T., Scheuermann, G.: Surface Techniques for Vortex Visualization. In: Proceedings of VisSym 2004, 155-164 (2004)

[GTS04] Garth, C., Tricoche, X., Scheuermann, G.: Tracking of Vector Field Singularities in Unstructured 3D Time-Dependent Datasets. In: Proc. IEEE Visualization 2004, 329-336 (2004)

[HH89] Helman, J.L., Hesselink, L.: Representation and Display of Vector Field Topology in Fluid Flow Data Sets, Computer, 22(8), 27-36 (1989).

[Hul92] Hultquist, J.P.M.: Constructing stream surfaces in steady 3D vector fields, In: Proceedings of the 3rd conference on Visualization '92, 171-178 (1992)

[LKG98] Löffelmann, H., Kucera, T., Gröller, E.: Visualizing Poincaré Maps Together With the Underlying Flow. In: Hege, H.C., Polthier, K. (eds.), Proceedings of the International Workshop on Visualization and Mathematics '97 315-328 (1998)

[LL99] de Leeuw, W., van Liere, R.: Collapsing flow topology using area metrics, In: Proc. IEEE Visualization 99, 149-354 (1999)

[MBS*04] Mahrous, K. Bennett, J., Scheuermann, G., Hamann, B. Joy, K.I.: Topological Segmentation in Three-Dimensional Vector Fields, IEEE Transactions on Visualization and Computer Graphics, 10(2), 198-205 (2004)

[SGH06] Surana, A., Grunberg, O., Haller, G.: Exact theory of threedimensional flow separation. Part I. Steady separation, J. Fluid Mech., 564, 57-103 (2006)

[SMH98] Spohn, A., Mory, M, Hopfinger, E.: Experiments on vortex breakdown in a confined flow generated by a rotating disk, J. Fluid Mech., 370, 73-99 (1998)

[SVL01] Sotiropoulos, F., Ventikos, Y., Lackey, T.: Chaotic advection in three-dimensional stationary vortex-breakdown bubbles: Sil'nikov's chaos and the devil's staircase, J. Fluid Mech., 444, 257-297 (2001)

[TH03] Thompson, M. Hourigan, K.: The sensitivity of steady vortex breakdown bubbles in confined cylinder flows to rotating lid misalignment, J. Fluid Mech., 496, 129138 (2003)

[TGK*04] Tricoche, X., Garth, C., Kindlmann, G.L., Deines, E., Scheuermann, G., Ruetten, M., Hansen, C.D.: Visualization of Intricate Flow Structures for Vortex Breakdown Analysis. In: Proceedings of IEEE Visualization '04, 187-194, 2004.

[TSH00] Tricoche, X., Scheuermann, G., Hagen, H.: A topology simplification method for 2D vector fields, In: Proc. IEEE Visualization 2000, 359-366 (2000)

[TWH*03] Theisel, H., Weinkauf, T., Hege, H.C., Seidel, H.P., Saddle Connectors - An Approach to Visualizing the Topological Skeleton of Complex 3D Vector Fields. In: Proc. IEEE Visualization 2003, 225-232 (2003)

[Ven02] Ventikos, Y.: The effect of imperfections on the emergence of three-dimensionality in stationary vortex breakdown bubbles, Physics of Fluids, L13-L16 (2002)

[WS02] Wischgoll, T., Scheuermann, G.: Locating closed streamlines in 3D vector fields, In: D. Ebert, P. Brunet, I. Navazo (eds.), Data Visualisation 2002, Eurographics Association, 227-232 (2002)

[YKP05] Ye, X., Kao, D., Pang, A., Strategy for Scalable Seeding of 3D Streamlines, In: Proceedings of IEEE Visualization '05, Minneapolis (2005) 